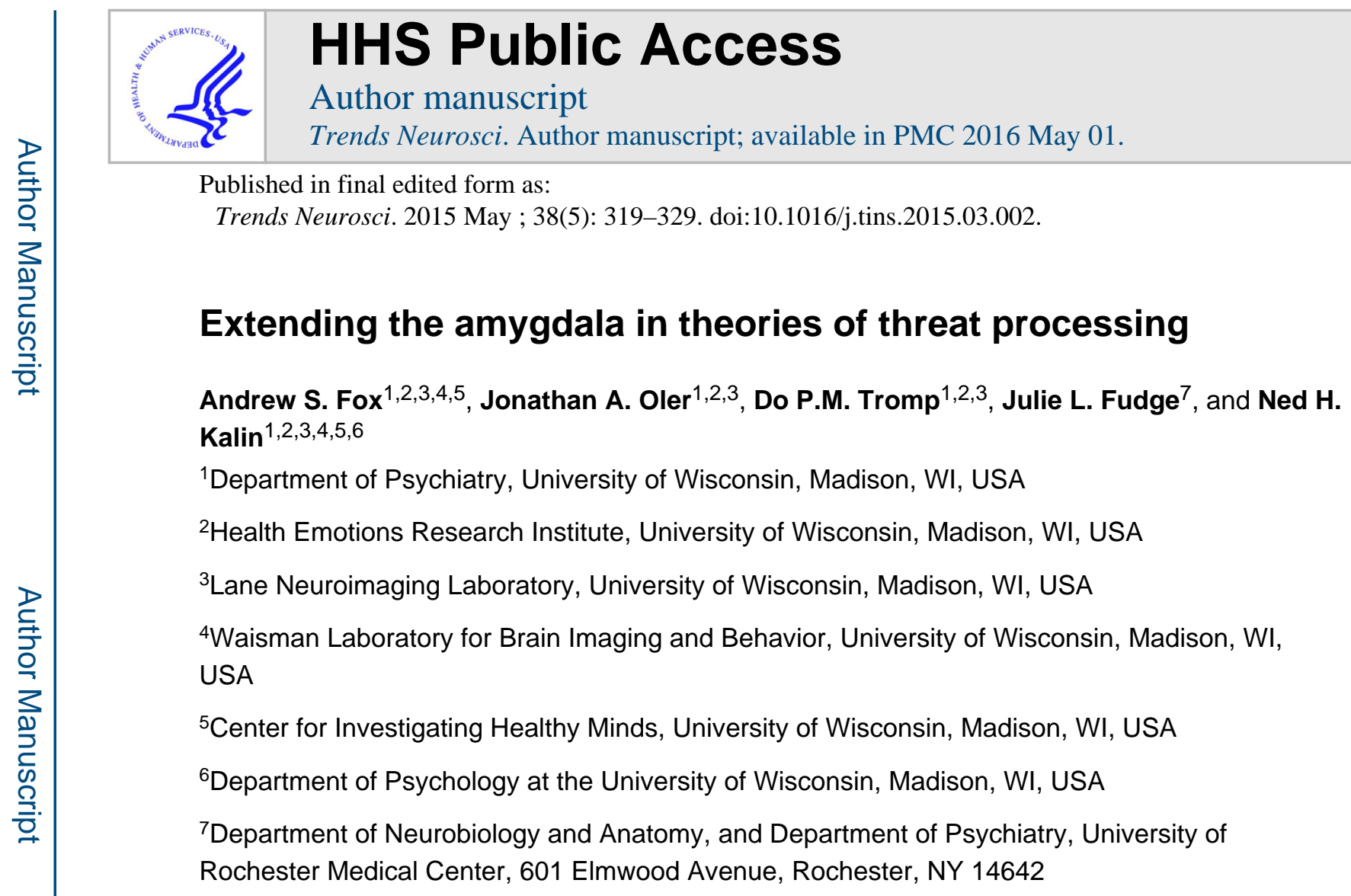

\title{
Abstract
}

The central extended amygdala is an evolutionarily conserved set of interconnected brain regions that play an important role in threat processing to promote survival. Two core components of the central extended amygdala, the central nucleus of the amygdala $(\mathrm{Ce})$ and the lateral bed nucleus of the stria terminalis (BST) are highly similar regions that serve complimentary roles by integrating fear- and anxiety-relevant information. Survival depends on the central extended amygdala's ability to rapidly integrate and respond to threats that vary in their immediacy, proximity, and characteristics. Future studies will benefit from understanding alterations in central extended amygdala function in relation to stress-related psychopathology.

\section{Extending the Amygdala}

The term amygdala refers to a group of subregions or nuclei that together comprise a core component of an emotion-related network. This anatomical concept has been of considerable value and is fundamental to theories of threat-processing and emotion dysfunction [1-5]. In large part, based on rodent studies of threat-processing, the amygdala has become a region of intense interest in psychiatric research focused on the pathogenesis of anxiety disorders and affective disorders [6,7]. Importantly, the amygdala subnuclei do not function in isolation. Their dense connectivity with other brain regions is critical for adaptively responding to stress as well as in facilitating responding to both explicitly cued

\footnotetext{
(C) 2015 Published by Elsevier Ltd.
}

Publisher's Disclaimer: This is a PDF file of an unedited manuscript that has been accepted for publication. As a service to our customers we are providing this early version of the manuscript. The manuscript will undergo copyediting, typesetting, and review of the resulting proof before it is published in its final citable form. Please note that during the production process errors may be discovered which could affect the content, and all legal disclaimers that apply to the journal pertain. 
immediate threats and distant threats that are more uncertain. It is notable that some of the amygdala's nuclei share similarities with other basal forebrain neurons, providing the basis for the concept of the "extended amygdala". Here we review animal research, and recent human neuroimaging studies, to highlight the extended amygdala, and its role in fear and anxiety [8-10]. Our intent is to encourage researchers interested in stress, emotion, and psychopathology to consider the concept of the extended amygdala and its intricate microcircuitry as they interpret their findings. Even though the tools available to human researchers currently cannot fully dissociate the extended amygdala from nearby regions, the concept of the extended amygdala is highly relevant to understanding the pathophysiology of anxiety disorders and depressive disorders (see Box 1).

First, we discuss the rationale for grouping regions into an extended amygdala circuit. We focus on two major components, one within the amygdala proper, the central nucleus (Ce), and the other outside the amygdala, within the bed nucleus of stria terminalis (BST). Next, we discuss the cross-species evidence linking Ce and BST to anxiety- and fear-related responding. Specifically, we examine how the proposed roles for Ce and BST in threat processing interact to give rise to fear- and anxiety-related behaviors that function to promote survival. Survival depends on interaction between extended amygdala regions to rapidly integrate and respond to threats that vary in their immediacy, proximity, and characteristics. The extended amygdala is an important and useful concept that will help further the understanding of adaptive and maladaptive expressions of fear and anxiety in humans.

\section{Anatomy of Ce and BST}

The amygdala is not a single functional or structural unit, rather it is composed of numerous subnuclei that have been suggested to constitute at least three different anatomical and functional networks [9](Fig 1). The olfactory network involves the medial nucleus of the amygdala (Me) connecting with structures involved in olfaction, including olfactory predator cues [11]. The frontotemporal network involves the large basal and lateral nuclei of the amygdala with their cortical connections. Most relevant to this review is the autonomic network which, via the $\mathrm{Ce}$, projects to brainstem and hypothalamic structures that are necessary to mount fear- and anxiety-related responses [1,9]. As early as 1923, Johnston noted similarities between the Ce and BST based on developmental and cross-species comparisons [12]. These observations, along with evidence of similar cell-types, dense interconnections between the structures, and similar connectivity patterns with other brain regions, led Alheid \& Heimer to propose a new anatomical concept termed the extended amygdala [8]. Their initial description of the extended amygdala highlighted the $\mathrm{Ce}$ and BST along with other primarily GABAergic regions that included: the intercalated masses interspersed throughout the amygdala; the medial nucleus of the amygdala; portions of the nucleus accumbens (NAcc) bordering the BST; and "cell corridors" throughout the white matter connecting the Ce and BST (e.g. substantia innominata). They further subdivided the extended amygdala into medial and central divisions. The medial extended amygdala, named for its inclusion of Me, also includes medial subdivisions of the BST. Whereas, the central extended amygdala is named for its inclusion of the $\mathrm{Ce}$, and also contains the lateral regions of the BST (for greater detail, see $[8,13,14]$ ). 
The remainder of this review will focus on the central extended amygdala because of its well-documented role in autonomic regulation and threat responding. It is notable that the core components of the central extended amygdala are even more complex, in that $\mathrm{Ce}$ and lateral BST are comprised of multiple sub-regions (see Fig 1). To be specific, the $\mathrm{Ce}$ contains the lateral $\mathrm{Ce}(\mathrm{CeL})$, medial $\mathrm{Ce}(\mathrm{CeM})$ and capsular $\mathrm{Ce}(\mathrm{CeC})$, and the central BST is comprised of the lateral BST sub-regions (See Fig 1). It is important to emphasize that the central extended amygdala is not alone in its capacity to initiate threat-responses, as there are other substrates capable of initiating threat-responding [15]. Moreover, the central extended amygdala is not uniquely associated with threat-processing, as it plays a role in other processes, including reward [16](see Box 2 for a brief discussion).

\section{Ce and BST have similar connectivity patterns}

The Ce and BST are thought to have generally similar patterns of efferent/afferent connectivity, however the degree of similarity has been a topic of substantial discussion $[9,17,18]$. With regard to their potenti involvement in threat processing, both Ce and BST project to threat-related brainstem and hypothalamic regions [9,19-21], and receive input from cortical regions, such as prefrontal and insular cortices [22-25]. To further examine similarities in connectivity of these structures, we drew upon a publicly available database from mouse tract tracing studies of brain-wide efferent and afferent connectivity [26]. As can be seen in Fig 2a-b, these analyses revealed that, in the mouse, the BST subnuclei have strikingly similar efferent and afferent connectivity patterns to those of the related $\mathrm{Ce}$ subnuclei.

Recent work using structural and functional neuroimaging of the human brain broadly supports the conclusions of rodent tract tracing studies [27,28]. For example, the C and BST interconnections via the ventral amygdalofugal pathway and the stria terminalis can be identified and quantified in vivo using diffusion tensor imaging (e.g. Fig 1). We used fMRI in young rhesus monkeys and adolescent humans to demonstrate that resting functional activation within Ce and BST is highly coordinated [29].

Together, these connectivity data, support the idea that the central extended amygdala is ideally suited to interface between cortical systems and the downstream brainstem effectors that are required to mount anxiety- and fear-related responses.

\section{Similarities in cellular composition between Ce and BST}

Heimer and colleague noted that neurons in the Ce and BST share similar morphological and neurochemical characteristics $[8,30]$. While there are multiple subtypes, in general the neurons of the central extended amygdala resemble GABAergic neurons found in striatal regions $[14,31,32]$. However, unlike the striatum, GABAergic neurons in Ce and BST coexpress various of neuropeptides [14,33]. Supporting their phenotypic similarities, recent studies reveal shared embryological origins of components of the central extended amygdala [34]. The Allen Institute for Brain Science (AIBS) mouse atlas allows for detailed analyses of regional patterns of gene expression [35]. Because gene expression is an intermediary between DNA and the proteins that determine cellular phenotypes, cells with similar origins and functions should, to some extent, share similar gene expression profiles [36,37]. As can 
be seen in Fig 2c, we found robust correlations between gene expression in subnuclei of the $\mathrm{Ce}$ with those of the BST. In general, the magnitude of Ce-BST correlations exceeded those between $\mathrm{Ce}$ and other regions. These gene expression findings provide additional and novel evidence supporting the relatedness of the major components of the central extended amygdala.

\section{Cross-species similarities and differences}

Primates diverged from rodents approximately 75 million years ago, and this divergence is reflected in the ontogenesis and refinement of neural systems, including a well-developed cortex. Evolutionary pressures affected the orientation of the amygdala, such that the rodent lateral surface is similar to the primate ventral surface (seen in Fig 1). Despite these species differences, initial studies suggest that the gross structure, cellular composition of amygdalar subnuclei, and many intra-amygdala connections are generally conserved. However, there are a number of important species differences. As compared to rodents that have a highly developed sense of smell, primates rely on visual and auditory information. As a result, human and nonhuman primates have evolved elaborate sensory association cortices, devoted to visual and auditory processes, as compared to the olfactory systems that are welldeveloped in the rodent $[38,39]$. These sensory differences between primates and rodents are also reflected in primates' relative enlargement of the basal and lateral amygdala nuclei that process incoming visual and auditory information [40,41]. In addition, in primates the basal and lateral amygdala nuclei have larger gradients in cell-size and relatively more glial cells [42]. Moreover, the paralaminar nucleus, a small-celled nucleus that in its ventral position envelops the basal and lateral nuclei, is prominent in primates but is not apparent in rodents [43]. Importantly, as compared to rodents, regions of the primate central extended amygdala are relatively enlarged compared to regions of the medial extended amygdala [44-46]. This enlargement is likely a reflection of the dense input that that Ce and BST receive from the primate-enlarged basal and lateral nuclei, along with a decreased dependence on olfactoryrelated information that is processed by the medial extended amygdala. Together these findings identify enough differences between rodent and primate extended amygdala that studies performed in rodents cannot be assumed to apply to the non-human primate. Nevertheless, the cross-species similarities provide a strong basis for forming translational hypotheses based on rodent work.

\section{Threat responsivity of the $\mathrm{Ce} \& \mathrm{BST}$}

Extensive studies examining sub-regions of the amygdala demonstrated involvement of different amygdala nuclei in mediating acquisition of, response to, and extinction of learned threats. In general the BLA is most related to the acquisition of conditioned threat, and the medial amygdala, as a component of the medial extended amygdala, has an important role in processing threat-related odors. The central extended amygdala, including the $\mathrm{Ce}$, is thought to provide an interface between the basal regions of the amygdala and the downstream targets required to initiate physiological, behavioral, and emotional responses. 


\section{Specific Functions of $\mathrm{Ce}$ and BST}

Studies demonstrate selective, but adaptively related, functions of Ce and BST in response to threat. Ce lesions produce deficits in threat responding in paradigms examining both conditioned and unconditioned threat [19]. Interestingly, BST lesions do not alter responses to threat in many of these same paradigms. Initial work by Davis and colleagues highlighted differences in function between Ce and BST. In rodents, BST lesions, but not Ce lesions, decreased the startle-enhancing effects of light, thought to reflect unconditioned threatresponses, and selectively reduced the startle enhancing effects of the anxiogenic peptide corticotrophin-releasing hormone (CRH)[47]. These and other studies showing a selective role for the BST in mediating responses to unconditioned and long-lasting stimuli led Davis and colleagues to initially propose that the Ce was responsible for "fear" whereas the BST was specifically related to "anxiety." In refining their view of the BST, Davis and colleagues suggested it is more appropriate to link the BST to maintaining prolonged threatpreparedness $[10,48]$. For example, BST lesions attenuate motivated actions during long (10min) but not short (1min) threat-cue exposure [49]. Moreover, pharmacological activation of BST using Calcitonin gene-related peptide (CGRP) increases unconditioned responses during exposure to potentially threatening contexts that require sustained vigilance [50,51]. Another study demonstrated that Ce lesions decreased freezing to an aversively conditioned cue, whereas BST lesions blocked the increased baseline freezing occurring after repeated threat-cue presentation [52]. BST lesions also attenuate the naturally occurring generalization of a threat-response to neutral cue presentation after cuedthreat conditioning [53].

These studies demonstrate that, at least in rodents, the central extended amygdala is critical for anxiety- and fear-related responding induced by threat. Mechanistic studies focused on central extended amygdala microcircuitry reveal that its functional organization is well suited to integrate evaluative and regulatory signals with sensory information to initiate behavioral and physiological responses to stress. Sensory information is primarily transmitted to the Ce from the basolateral amygdala (BLA), but hippocampus and prefrontal cortex can also influence Ce. For example, prefrontal inputs can modulate Ce via intermediate GABAergic neurons in the intercalated cell islands [54,55]. The $\mathrm{Ce}$ then integrates these regulatory/evaluative inputs to initiate coordinated emotional responses. Within the $\mathrm{Ce}$, the $\mathrm{CeM}$ contains neurons that project to brainstem structures that mediate specific behavioral and physiological responses to threat [56,57]. These outputs can be coordinated, and modulated by inhibitory CeL networks to produce context appropriate coordinated responses [56,58-60]. Recent work in rodents is beginning to identify a similar functional organization within the BST, such that the CeL-like BSTov modulates function in the CeM-like BSTal, which, like the CeM projects to downstream regions that mediate threat responsivity [61]. Through these Ce and BST microcircuits the central extended amygdala gates sensory and prefrontal triggers of threat responding, facilitating the regulation of anxiety- and fear- related behavior by integrating sources of "bottom-up" and "top-down" information. 


\section{Integrative functions of the $\mathrm{Ce}$ and BST}

Microcircuitry studies of the amygdala and extended amygdala in rodents provide a framework for understanding how the sub-nuclei of these structures complexly interact to support adaptive responses. It is likely that the selective functions ascribed to Ce and BST work in concert to promote survival. Alterations in the functions of these structures may conspire to disrupt adaptive responding in individuals suffering from anxiety disorders and other stress-related psychopathology. In real life, direct threats often occur within contexts that require safety monitoring and vigilance. Thus, although Ce and BST functions may be dissociable, Ce-related immediate self-preservation responses to threat often co-occur with BST-related sustained preparedness.

Our work in non-human primates, recently reviewed elsewhere [62,63], has identified that both Ce and BST metabolism are associated with freezing during sustained exposure to a potentially threatening human predator. We have examined hundreds of animals using the human intruder paradigm, in which a human presents their profile and makes no-eye-contact (NEC) with the monkey. Unlike more directly threatening contexts, such as when a human intruder stares at the animal, the uncertain and potentially-threatening NEC context elicits a robust avoidant and defensive behavioral response [64]. During NEC, animals, on average, increase their freezing, emit fewer vocalizations, and have increased activation of the HPA axis. Interestingly, there is large individual variation in behavior, such that during the NEC context extreme animals freeze for the entire 30-minutes of NEC exposure, while others may not freeze at all. Examining this natural variation in NEC-elicited freezing in relation to brain metabolism using FDG-PET, we demonstrated that individual differences in freezing, cooing, and cortisol are associated with variation in both Ce (verified with chemoarchitectonic imaging of the serotonin transporter) and BST-region metabolism [6570]. Because the FDG-PET data reflects integrated metabolism over a 30-minute uptake period, measurements of metabolic activity during the NEC context captures, and does not differentiate between, acute and sustained components of the response to threat.

We use the term anxious temperament (AT) to describe trait-like individual differences in the behavioral and physiological responses to the NEC context (i.e. increased freezing, decreased coo calling, and increased cortisol secretion). We have demonstrated that AT is a trait-like measure that is stable across both time and context [66,71]. Moreover, we found that brain metabolism associated with individual differences in AT is also stable across time and context [66,71]. In a study of 238 young rhesus monkeys phenotyped for NEC-induced AT and brain metabolism, we demonstrated that AT was associated with increased metabolism in diverse brain regions, including clusters which encompass central extended amygdala regions [69]. Consistent with these results, Ce lesions decreased freezing and increased affinitive vocalizations during exposure to the potentially threatening NEC context [72]. Interestingly, lesions of the whole-amygdala did not alter the same threat-related behaviors and, though more study is needed to verify this result, it raises the possibility of opposing or compensatory mechanisms within or outside of the amygdala [73].

In addition to identifying central extended amygdala regions, our studies in non-human primates have revealed a larger network of brain regions that are involved in threatresponding, including the brainstem, insula, and orbitofrontal cortex regions. Interestingly, 
nearly all of these regions are connected to, and interact with the Ce and BST. This led us to speculate that the effects of OFC lesions in reducing freezing behavior [74-76] could be mediated by the central extended amygdala. In a combined lesion and imaging study, we found that OFC lesions decreased NEC-induced freezing, along with metabolism in the BST-region during NEC [77]. Moreover, in this same study, BST-region metabolism was correlated with freezing behavior both pre- and post-lesion, suggesting that the BST might mediate the effects of OFC lesions on anxiety-related behavior. These data highlight the role of the central extended amygdala as a neural hub in integrating threat-related contextual information to coordinate the magnitude and quality of expressed defensive responses to threat.

\section{The human central extended amygdala in fear, anxiety, and anxiety disorders}

Much of the human neuroimaging work on fear, anxiety, and anxiety disorders has focused on the amygdala per se, with fewer studies attempting to parse the functions of its subnuclei. Nevertheless, a substantial number of reports identify threat-related activation in the dorsal amygdala region that encompasses the Ce [19,78]. Recent human fMRI studies have tested BST-focused hypotheses derived from the animal literature, identifying contexts that elicit BST activation. Future studies focused on the central extended amygdala can be informed by a more detailed examination of these initial studies. In one BST focused study, healthy young adults were shown a fake trace of their physiological response (i.e. skin conductance response; SCR), and were told they would receive a shock each time their fake-SCR reached a certain threshold [79]. BST-region activation increased as participants observed their fakeSCR approach the shock-threshold (Fig 3b, left). In another study of healthy subjects, increased BST-region activation occurred when subjects perceived a spider advancing toward their foot [80](Fig 3b, right). Similarly, when subjects played a pac-man like videogame where capture was associated with an electric shock, BST-activation increased as the computer closed in on the subject's avatar [81]. Increased BST-region activation has also been observed while healthy participants contemplate dangerous situations, such as the likelihood of highly harmful events, e.g. breaking several ribs, drowning, or paralysis [82]. In other studies, the BST-region has been identified as showing sustained activation during anticipation of uncertain negative events. For example, sustained BST-region activation occurred during threat-eliciting blocks of negative pictures when the time of picture presentation was unpredictable [83]; while participants awaited the unpredictable onset of a negative image [84]; and, during exposure to a context in which a shock might, or might not, occur [85-87]. Consistent with our OFC-lesion studies in non-human primates [77], a recent study found that patients with ventromedial prefrontal cortex damage had decreased resting blood flow in the BST-region, consistent with a role for prefrontal regulation of BSTfunction [88]. Importantly, data from attempts to dissociate anxiety-related activation of $\mathrm{Ce}$ from BST by Grillon and colleagues are largely consistent with the animal work, and are beginning to selectively implicate the BST in long-term threat responses and threat preparedness [48]. Together, these data provide the foundation for human studies aimed at understanding the role of the central extended amygdala in threat processing. 
Although few publications discuss the BST by name, meta-analyses of activation patterns from these and other reports implicate the BST-region in the human experience of fear and anxiety. This is the case for a meta-analysis examining the neural substrates underlying the experience of negative affect, as well as those examining threat conditioning [89,90] (http:// www.columbia.edu/cu/psychology/tor/MetaAnalysis.htm; Fig. 3c). A meta-analysis of neuroimaging studies using instructed threat paradigms, though never mentioning BST, reported an activation on the midline just superior to the anterior commissure consistent with the location of the BST [91]. In addition, an automated meta-analysis using Neurosynth [92] found that the 222 brain imaging papers using the word "anxiety" were more likely to report activations in coordinates near the BST in comparison to all of the papers in the database (Fig 3d; similar results for "fear" can be found at Neurosynth.org). These data suggest that activation in coordinates associated with the BST-region are frequently included in reports, but are not explicitly referred to as BST. It is possible that activations stemming from the BST region have been attributed to bordering regions, such as the caudate $[93,94]$ and nucleus accumbens [95]. The results of the relatively few BST-focused articles, along with the above coordinate-based meta-analyses, suggest that the BST, a key component of the central extended amygdala, plays an important role in threat processing in humans.

There are a few studies that have focused on BST-alterations in anxiety disordered patient populations, and they report increased BST-region activation. For example, increased BSTregion activation was noted in spider-phobics during anticipation of phobia relevant images, in patients with post-traumatic stress disorder (PTSD) looking at combat-relevant images [96]; and, in patients with generalized anxiety disorder (GAD) during a gambling task [97]. These data point to the BST as a region that plays a role in the suffering of patients with anxiety disorders, and provide an impetus for further study of BST in relation to stressrelated psychopathology.

\section{Opportunity and New Frontier}

The historical evidence, along with recent large-scale data based analyses at the molecular and systems levels, convincingly support the concept of the central extended amygdala. As key components of the central extended amygdala, these approaches also establish the similarity of structure and function between the Ce and BST. Based on animal and human studies, there is no question that the central extended amygdala is involved in adaptive and maladaptive threat responding. Nevertheless, much work needs to be done. Although animal research has begun to elucidate the role of the Ce and BST, it is important to remember that the central extended amygdala has other components (e.g. substantia innominata) that, with further investigation, may be found to play a critical role in threat-processing and preparedness. For reasons already discussed in this review (see Box 1), there are a paucity of human neuroimaging studies that have explicitly focused on the BST-region. However, meta-analyses of human neuroimaging studies provide compelling evidence that the BSTregion is associated with fear and anxiety in humans. Thus, because of the potential importance of the BST, it is critical for researchers to incorporate the proposed function of the central extended amygdala into their theories of adaptive and maladaptive threat processing, and to design human studies that optimally reveal threat-related activations in the central extended amygdala. 
The central extended amygdala mediates the adaptive responding to perceived threat, as the central extended amygdala is an interface between vigilance, internal emotional states, and associated physiological and behavioral responses. Components of this interface include the $\mathrm{Ce}$ and BST, each of which have been demonstrated, under certain conditions, to have dissociable roles in mediating the expression of threat responding. It is likely that a more refined understanding of the overlaps and differences in the function of these structures will provide insight into the wide range of subjective experiences associated with negative affect, fear, and anxiety. Because of the marked connectivity between Ce and BST, there will be significant value in understanding the integrated function of components of the central extended amygdala. Given what we know about the Ce and BST from mechanistic animal work, it is hard to imagine real-life situations involving threat that depend solely on the actions of a single extended amygdala subregion. Imagine yourself walking alone at dusk in an unknown wooded area, while already feeling wary, you jump at the sight of a snake, which further increases your apprehension as darkness ensues. Here, the subjective experience of wariness and apprehension as a consequence of a potential threat requires BST-mediated sustained threat-preparedness to interact with Ce-mediated acute threat responding. The evolutionary pressures to survive in the face of potential predation are so strong that a major function of the brain is to continuously process and scan the environment to enable rapid and successful defensive responding. In this regard, the central extended amygdala, as a threat detector and effector, is critical for survival. By continuously functioning in the background as a mechanism to rapidly and reliably respond to potential danger, the central extended amygdala enables organisms to feel secure in exploring new environments and engaging in appetitive behaviors. Thus, excessive activity within central extended amygdala circuits is likely to bring fears to the forefront, tipping the balance between appetitive and defensive behavior resulting in excessive and inappropriate threat responding. This threat-related shift from positive to negative affect, associated with avoidance and withdrawal, is pathognomonic of anxiety disorders and depressive disorders. To develop novel treatments aimed at preventing and ameliorating the symptoms of severe anxiety and fear, it is imperative to further elucidate the role of the extended amygdala in mediating anxiety disorders and stress-related psychopathology.

\section{Acknowledgments}

The authors thank P.H. Roseboom, R. Kovner, L.E. Williams, and A.J. Shackman for their insights and discussions surrounding the extended amygdala, along with the dedicated staff of the Harlow Center for Biological Psychology at the University of Wisconsin-Madison. The authors were supported by the National Institutes of Health (NIH; Intramural Research Program and extramural grants R21MH91550, R01MH81884, R01MH46729, P50MH84051, P50MH100031, R21MH092581), and the HealthEmotions Research Institute. Authors declare no conflicts of interest.

\section{References}

1. Adolphs R. The biology of fear. Curr Biol. 2013; 23:R79-93. [PubMed: 23347946]

2. Ledoux, J. The Emotional Brain: The Mysterious Underpinnings of Emotional Life. Simon and Schuster; 1998.

3. Lindquist KA, et al. The brain basis of emotion: a meta-analytic review. Behav Brain Sci. 2012; 35:121-143. [PubMed: 22617651]

4. Paré D, et al. New vistas on amygdala networks in conditioned fear. J Neurophysiol. 2004; 92:1-9. [PubMed: 15212433] 
5. Phelps EA. Emotion and cognition: insights from studies of the human amygdala. Annu Rev Psychol. 2006; 57:27-53. [PubMed: 16318588]

6. Rauch SL, et al. Neuroimaging studies of amygdala function in anxiety disorders. Ann N Y Acad Sci. 2003; 985:389-410. [PubMed: 12724173]

7. Ressler KJ, Mayberg HS. Targeting abnormal neural circuits in mood and anxiety disorders: from the laboratory to the clinic. Nat Neurosci. 2007; 10:1116-1124. [PubMed: 17726478]

8. Alheid GF, Heimer L. New perspectives in basal forebrain organization of special relevance for neuropsychiatric disorders: the striatopallidal, amygdaloid, and corticopetal components of substantia innominata. Neuroscience. 1988; 27:1-39. [PubMed: 3059226]

9. Swanson LW, Petrovich GD. What is the amygdala? Trends Neurosci. 1998; 21:323-331. [PubMed: 9720596]

10. Walker DL, et al. Role of the bed nucleus of the stria terminalis versus the amygdala in fear, stress, and anxiety. Eur J Pharmacol. 2003; 463:199-216. [PubMed: 12600711]

11. Takahashi LK. Olfactory systems and neural circuits that modulate predator odor fear. Front Behav Neurosci. 2014; 8:72. [PubMed: 24653685]

12. Johnston JB. Further contributions to the study of the evolution of the forebrain. The Journal of Comparative Neurology. 1923; 35:337-481.

13. Dong HW, et al. Topography of projections from amygdala to bed nuclei of the stria terminalis. Brain Res Brain Res Rev. 2001; 38:192-246. [PubMed: 11750933]

14. Yilmazer-Hanke, DM. Amygdala. In: Mai, JK.; Paxinos, G., editors. The human nervous system. 3rd. Elsevier Academic Press; 2012. p. 759-834.

15. Gross CT, Canteras NS. The many paths to fear. Nat Rev Neurosci. 2012; 13:651-658. [PubMed: 22850830]

16. Everitt BJ, et al. Associative processes in addiction and reward. The role of amygdala-ventral striatal subsystems. Ann N Y Acad Sci. 1999; 877:412-438. [PubMed: 10415662]

17. McDonald AJ. Is there an amygdala and how far does it extend? An anatomical perspective. Ann $\mathrm{N}$ Y Acad Sci. 2003; 985:1-21. [PubMed: 12724144]

18. Zahm DS. Is the caudomedial shell of the nucleus accumbens part of the extended amygdala? A consideration of connections. Crit Rev Neurobiol. 1998; 12:245-265. [PubMed: 9847057]

19. Davis M, Whalen PJ. The amygdala: vigilance and emotion. Mol Psychiatry. 2001; 6:13-34. [PubMed: 11244481]

20. Dong HW, et al. Basic organization of projections from the oval and fusiform nuclei of the bed nuclei of the stria terminalis in adult rat brain. J Comp Neurol. 2001; 436:430-455. [PubMed: 11447588]

21. Dong HW, Swanson LW. Organization of axonal projections from the anterolateral area of the bed nuclei of the stria terminalis. J Comp Neurol. 2004; 468:277-298. [PubMed: 14648685]

22. Ferry AT, et al. Prefrontal cortical projections to the striatum in macaque monkeys: evidence for an organization related to prefrontal networks. J Comp Neurol. 2000; 425:447-470. [PubMed: 10972944]

23. Freedman LJ, et al. Subcortical projections of area 25 (subgenual cortex) of the macaque monkey. J Comp Neurol. 2000; 421:172-188. [PubMed: 10813780]

24. McDonald AJ, et al. Cortical afferents to the extended amygdala. Ann N Y Acad Sci. 1999; 877:309-338. [PubMed: 10415657]

25. Saper CB. Convergence of autonomic and limbic connections in the insular cortex of the rat. J Comp Neurol. 1982; 210:163-173. [PubMed: 7130477]

26. Bota M, Swanson LW. Online workbenches for neural network connections. J Comp Neurol. 2007; 500:807-814. [PubMed: 17177264]

27. Avery SN, et al. BNST neurocircuitry in humans. Neuroimage. 2014; 91:311-323. [PubMed: 24444996]

28. Roy AK, et al. Functional connectivity of the human amygdala using resting state fMRI. Neuroimage. 2009; 45:614-626. [PubMed: 19110061]

29. Oler JA, et al. Evidence for coordinated functional activity within the extended amygdala of nonhuman and human primates. NeuroImage. 201210.1016/j.neuroimage.2012.03.045 
30. De Olmos JS, Heimer L. The concepts of the ventral striatopallidal system and extended amygdala. Ann N Y Acad Sci. 1999; 877:1-32. [PubMed: 10415640]

31. McDonald AJ. Cytoarchitecture of the central amygdaloid nucleus of the rat. J Comp Neurol. 1982; 208:401-418. [PubMed: 7119168]

32. McDonald AJ. Neurons of the bed nucleus of the stria terminalis: a golgi study in the rat. Brain Res Bull. 1983; 10:111-120. [PubMed: 6824959]

33. Gray TS, Magnuson DJ. Peptide immunoreactive neurons in the amygdala and the bed nucleus of the stria terminalis project to the midbrain central gray in the rat. Peptides. 1992; 13:451-460. [PubMed: 1381826]

34. Bupesh M, et al. Genetic and experimental evidence supports the continuum of the central extended amygdala and a mutiple embryonic origin of its principal neurons. J Comp Neurol. 2011; 519:3507-3531. [PubMed: 21800302]

35. Lein ES, et al. Genome-wide atlas of gene expression in the adult mouse brain. Nature. 2007; 445:168-176. [PubMed: 17151600]

36. Grange $\mathrm{P}$, et al. Cell-type-based model explaining coexpression patterns of genes in the brain. Proc Natl Acad Sci USA. 2014; 111:5397-5402. [PubMed: 24706869]

37. $\mathrm{Ng} \mathrm{L}$, et al. An anatomic gene expression atlas of the adult mouse brain. Nat Neurosci. 2009; 12:356-362. [PubMed: 19219037]

38. Preuss TM, et al. Distinctive compartmental organization of human primary visual cortex. Proc Natl Acad Sci USA. 1999; 96:11601-11606. [PubMed: 10500223]

39. Yellin AM, Jerison HJ. Visual evoked potentials and inter-stimulus intervals in the rat. Electroencephalogr Clin Neurophysiol. 1973; 34:429-432. [PubMed: 4120317]

40. Pitkänen A, Amaral DG. Organization of the intrinsic connections of the monkey amygdaloid complex: projections originating in the lateral nucleus. J Comp Neurol. 1998; 398:431-458. [PubMed: 9714153]

41. Turner BH, et al. Organization of the amygdalopetal projections from modality-specific cortical association areas in the monkey. J Comp Neurol. 1980; 191:515-543. [PubMed: 7419732]

42. Chareyron LJ, et al. Stereological analysis of the rat and monkey amygdala. J Comp Neurol. 2011; 519:3218-3239. [PubMed: 21618234]

43. deCampo DM, Fudge JL. Where and what is the paralaminar nucleus? A review on a unique and frequently overlooked area of the primate amygdala. Neurosci Biobehav Rev. 2012; 36:520-535. [PubMed: 21906624]

44. Andy OJ, Stephan H. The septum in the human brain. J Comp Neurol. 1968; 133:383-410. [PubMed: 5710964]

45. Price, JL., et al. The limbic region. II. The amygdaloid complex. In: Hokfelt, B.; Swanson, LW., editors. Handbook of Chemical Neuroanatomy. Elsevier; Amsterdam: 1987. p. 279-381.

46. Stephan H, Andy OJ. Quantitative comparison of the amygdala in insectivores and primates. Acta Anat (Basel). 1977; 98:130-153. [PubMed: 404833]

47. Davis M, et al. Amygdala and bed nucleus of the stria terminalis: differential roles in fear and anxiety measured with the acoustic startle reflex. Philos Trans R Soc Lond, B, Biol Sci. 1997; 352:1675-1687. [PubMed: 9415919]

48. Davis M, et al. Phasic vs sustained fear in rats and humans: role of the extended amygdala in fear vs anxiety. Neuropsychopharmacology. 2010; 35:105-135. [PubMed: 19693004]

49. Waddell J, et al. Effects of bed nucleus of the stria terminalis lesions on conditioned anxiety: aversive conditioning with long-duration conditional stimuli and reinstatement of extinguished fear. Behav Neurosci. 2006; 120:324-336. [PubMed: 16719697]

50. Sink KS, et al. Calcitonin gene-related peptide in the bed nucleus of the stria terminalis produces an anxiety-like pattern of behavior and increases neural activation in anxiety-related structures. $\mathrm{J}$ Neurosci. 2011; 31:1802-1810. [PubMed: 21289190]

51. Sink KS, et al. CGRP antagonist infused into the bed nucleus of the stria terminalis impairs the acquisition and expression of context but not discretely cued fear. Learn Mem. 2013; 20:730-739. [PubMed: 24255102] 
52. Walker DL, Davis M. Quantifying fear potentiated startle using absolute versus proportional increase scoring methods: implications for the neurocircuitry of fear and anxiety. Psychopharmacology (Berl). 2002; 164:318-328. [PubMed: 12424556]

53. Duvarci $S$, et al. The bed nucleus of the stria terminalis mediates inter-individual variations in anxiety and fear. J Neurosci. 2009; 29:10357-10361. [PubMed: 19692610]

54. Likhtik E, et al. Amygdala intercalated neurons are required for expression of fear extinction. Nature. 2008; 454:642-645. [PubMed: 18615014]

55. Royer S, et al. An inhibitory interface gates impulse traffic between the input and output stations of the amygdala. J Neurosci. 1999; 19:10575-10583. [PubMed: 10575053]

56. Ciocchi S, et al. Encoding of conditioned fear in central amygdala inhibitory circuits. Nature. 2010; 468:277-282. [PubMed: 21068837]

57. Viviani D, et al. Oxytocin selectively gates fear responses through distinct outputs from the central amygdala. Science. 2011; 333:104-107. [PubMed: 21719680]

58. Haubensak W, et al. Genetic dissection of an amygdala microcircuit that gates conditioned fear. Nature. 2010; 468:270-276. [PubMed: 21068836]

59. Pape HC, Pare D. Plastic synaptic networks of the amygdala for the acquisition, expression, and extinction of conditioned fear. Physiol Rev. 2010; 90:419-463. [PubMed: 20393190]

60. Pare D, Duvarci S. Amygdala microcircuits mediating fear expression and extinction. Current opinion in neurobiology. 201210.1016/j.conb.2012.02.014

61. Kim SY, et al. Diverging neural pathways assemble a behavioural state from separable features in anxiety. Nature. 2013; 496:219-223. [PubMed: 23515158]

62. Fox AS, Kalin NH. A Translational Neuroscience Approach to Understanding the Development of Social Anxiety Disorder and Its Pathophysiology. Am J Psychiatry. 201410.1176/appi.ajp. 2014.14040449

63. Oler, JA., et al. The central nucleus of the amygdala is a critical substrate for individual differences in anxiety. To appear in Living without an amygdala. In: Amaral, DG., editor. Living without an amygdala. Guilford Press;

64. Kalin NH, Shelton SE. Defensive behaviors in infant rhesus monkeys: environmental cues and neurochemical regulation. Science. 1989; 243:1718-1721. [PubMed: 2564702]

65. Fox AS, et al. Calling for help is independently modulated by brain systems underlying goaldirected behavior and threat perception. Proceedings of the National Academy of Sciences of the United States of America. 2005; 102:4176-4179. [PubMed: 15753316]

66. Fox AS, et al. Trait-Like Brain Activity during Adolescence Predicts Anxious Temperament in Primates. PLoS ONE. 2008; 3

67. Kalin NH, et al. Brain regions associated with the expression and contextual regulation of anxiety in primates. Biol Psychiatry. 2005; 58:796-804. [PubMed: 16043132]

68. Oler JA, et al. Serotonin transporter availability in the amygdala and bed nucleus of the stria terminalis predicts anxious temperament and brain glucose metabolic activity. J Neurosci. 2009; 29:9961-9966. [PubMed: 19675230]

69. Oler JA, et al. Amygdalar and hippocampal substrates of anxious temperament differ in their heritability. Nature. 2010; 466:864-868. [PubMed: 20703306]

70. Shackman AJ, et al. Neural mechanisms underlying heterogeneity in the presentation of anxious temperament. Proc Natl Acad Sci USA. 2013; 110:6145-6150. [PubMed: 23538303]

71. Fox AS, et al. Central amygdala nucleus (Ce) gene expression linked to increased trait-like Ce metabolism and anxious temperament in young primates. Proc Natl Acad Sci USA. 2012; 109:18108-18113. [PubMed: 23071305]

72. Kalin NH, et al. The role of the central nucleus of the amygdala in mediating fear and anxiety in the primate. J Neurosci. 2004; 24:5506-5515. [PubMed: 15201323]

73. Kalin NH, et al. The primate amygdala mediates acute fear but not the behavioral and physiological components of anxious temperament. J Neurosci. 2001; 21:2067-2074. [PubMed: 11245690]

74. Kalin NH, et al. Role of the primate orbitofrontal cortex in mediating anxious temperament. Biol Psychiatry. 2007; 62:1134-1139. [PubMed: 17643397] 
75. Machado CJ, Bachevalier J. Behavioral and hormonal reactivity to threat: effects of selective amygdala, hippocampal or orbital frontal lesions in monkeys. Psychoneuroendocrinology. 2008; 33:926-941. [PubMed: 18650022]

76. Murray EA, Izquierdo A. Orbitofrontal cortex and amygdala contributions to affect and action in primates. Ann N Y Acad Sci. 2007; 1121:273-296. [PubMed: 17846154]

77. Fox AS, et al. Orbitofrontal cortex lesions alter anxiety-related activity in the primate bed nucleus of stria terminalis. J Neurosci. 2010; 30:7023-7027. [PubMed: 20484644]

78. Whalen PJ, et al. A functional MRI study of human amygdala responses to facial expressions of fear versus anger. Emotion. 2001; 1:70-83. [PubMed: 12894812]

79. Somerville LH, et al. Human bed nucleus of the stria terminalis indexes hypervigilant threat monitoring. Biol Psychiatry. 2010; 68:416-424. [PubMed: 20497902]

80. Mobbs D, et al. Neural activity associated with monitoring the oscillating threat value of a tarantula. Proc Natl Acad Sci USA. 2010; 107:20582-20586. [PubMed: 21059963]

81. Mobbs D, et al. When fear is near: threat imminence elicits prefrontal-periaqueductal gray shifts in humans. Science. 2007; 317:1079-1083. [PubMed: 17717184]

82. Coaster M, et al. Variables influencing the neural correlates of perceived risk of physical harm. Cogn Affect Behav Neurosci. 2011; 11:494-507. [PubMed: 21671045]

83. Somerville LH, et al. Interactions Between Transient and Sustained Neural Signals Support the Generation and Regulation of Anxious Emotion. Cerebral Cortex (New York, NY: 1991). 201210.1093/cercor/bhr373

84. Grupe DW, et al. Dissecting the anticipation of aversion reveals dissociable neural networks. Cereb Cortex. 2013; 23:1874-1883. [PubMed: 22763169]

85. Alvarez RP, et al. Phasic and sustained fear in humans elicits distinct patterns of brain activity. Neuroimage. 2011; 55:389-400. [PubMed: 21111828]

86. Choi JM, et al. Impact of state anxiety on the interaction between threat monitoring and cognition. Neuroimage. 2012; 59:1912-1923. [PubMed: 21939773]

87. Klumpers F, et al. Dorsomedial Prefrontal Cortex Mediates the Impact of Serotonin Transporter Linked Polymorphic Region Genotype on Anticipatory Threat Reactions. Biol Psychiatry. 201410.1016/j.biopsych.2014.07.034

88. Motzkin JC, et al. Ventromedial prefrontal cortex damage alters resting blood flow to the bed nucleus of stria terminalis. Cortex. 2014; 64C:281-288. [PubMed: 25569763]

89. Etkin A, Wager TD. Functional neuroimaging of anxiety: a meta-analysis of emotional processing in PTSD, social anxiety disorder, and specific phobia. Am J Psychiatry. 2007; 164:1476-1488. [PubMed: 17898336]

90. Lewis, M., et al., editors. Handbook of Emotions, Third Edition. Third. The Guilford Press; 2008.

91. Mechias ML, et al. A meta-analysis of instructed fear studies: implications for conscious appraisal of threat. Neuroimage. 2010; 49:1760-1768. [PubMed: 19786103]

92. Yarkoni T, et al. Large-scale automated synthesis of human functional neuroimaging data. Nat Methods. 2011; 8:665-670. [PubMed: 21706013]

93. Coan JA, et al. Lending a hand: social regulation of the neural response to threat. Psychol Sci. 2006; 17:1032-1039. [PubMed: 17201784]

94. Dunsmoor JE, et al. Neurobehavioral mechanisms of human fear generalization. Neuroimage. 2011; 55:1878-1888. [PubMed: 21256233]

95. Levita L, et al. Avoidance of harm and anxiety: a role for the nucleus accumbens. Neuroimage. 2012; 62:189-198. [PubMed: 22569544]

96. Morey RA, et al. The role of trauma-related distractors on neural systems for working memory and emotion processing in posttraumatic stress disorder. J Psychiatr Res. 2009; 43:809-817. [PubMed: 19091328]

97. Yassa MA, et al. Functional MRI of the amygdala and bed nucleus of the stria terminalis during conditions of uncertainty in generalized anxiety disorder. J Psychiatr Res. 2012; 46:1045-1052. [PubMed: 22575329]

98. Holland, null; Gallagher, null. Amygdala circuitry in attentional and representational processes. Trends Cogn Sci (Regul Ed). 1999; 3:65-73. [PubMed: 10234229] 
99. Comoli E, et al. Functional mapping of the prosencephalic systems involved in organizing predatory behavior in rats. Neuroscience. 2005; 130:1055-1067. [PubMed: 15653000]

100. Knapska E, et al. Functional internal complexity of amygdala: focus on gene activity mapping after behavioral training and drugs of abuse. Physiol Rev. 2007; 87:1113-1173. [PubMed: 17928582]

101. Jennings JH, et al. Distinct extended amygdala circuits for divergent motivational states. Nature. 2013; 496:224-228. [PubMed: 23515155]

102. Choi JM, et al. Pervasive competition between threat and reward in the brain. Soc Cogn Affect Neurosci. 2014; 9:737-750. [PubMed: 23547242]

103. Reynolds SM, Berridge KC. Emotional environments retune the valence of appetitive versus fearful functions in nucleus accumbens. Nat Neurosci. 2008; 11:423-425. [PubMed: 18344996]

104. Gabriel M, et al. Consideration of a unified model of amygdalar associative functions. Ann N Y Acad Sci. 2003; 985:206-217. [PubMed: 12724160]

105. Stamatakis AM, et al. Amygdala and bed nucleus of the stria terminalis circuitry: Implications for addiction-related behaviors. Neuropharmacology. 2014; 76 Pt B:320-328. [PubMed: 23752096]

106. Canteras NS, et al. Organization of projections from the medial nucleus of the amygdala: a PHAL study in the rat. J Comp Neurol. 1995; 360:213-245. [PubMed: 8522644]

107. Kemble ED, et al. Taming in wild rats following medial amygdaloid lesions. Physiol Behav. 1984; 32:131-134. [PubMed: 6538976]

108. Roseboom PH, et al. Predator threat induces behavioral inhibition, pituitary-adrenal activation and changes in amygdala CRF-binding protein gene expression. Psychoneuroendocrinology. 2007; 32:44-55. [PubMed: 17116372]

109. Martinez RC, et al. Amygdalar roles during exposure to a live predator and to a predatorassociated context. Neuroscience. 2011; 172:314-328. [PubMed: 20955766]

110. Wallis JD. Cross-species studies of orbitofrontal cortex and value-based decision-making. Nat Neurosci. 2012; 15:13-19. [PubMed: 22101646]

111. Buhle JT, et al. Cognitive Reappraisal of Emotion: A Meta-Analysis of Human Neuroimaging Studies. Cereb Cortex. 201310.1093/cercor/bht154

112. Wilent WB, et al. Induction of panic attack by stimulation of the ventromedial hypothalamus. $\mathbf{J}$ Neurosurg. 2010; 112:1295-1298. [PubMed: 19852539]

113. Nashold BS Jr, et al. Sensations evoked by stimulation in the midbrain of man. J Neurosurg. 1969; 30:14-24. [PubMed: 4885810]

114. McMenamin BW, et al. Network organization unfolds over time during periods of anxious anticipation. J Neurosci. 2014; 34:11261-11273. [PubMed: 25143607]

115. Mai, JK., et al. Atlas of the Human Brain, Third Edition. 3. Academic Press; 2007.

116. Paxinos, G. The rhesus monkey brain in stereotaxic coordinates. 2nd. Academic; 2009.

117. Heimer, L., et al. Chapter II The human basal forebrain. Part II. In: Bloom, FE.; B, A.; H, T., editors. Handbook of Chemical Neuroanatomy. Vol. 15. Elsevier; 1999. p. 57-226. 


\section{Highlights}

- Central extended amygdala includes portions of the amygdala $(\mathrm{Ce})$ and lateral BST.

- Central extended amygdala regions share connectivity and gene expression patterns.

- Animal studies show central extended amygdala to be involved in fear and anxiety.

- Human functional imaging studies are beginning to translate these animal findings.

- Maladaptive central extended amygdala function may underlie stress-related psychopathology. 


\section{BOX 1: Citing the BST in human fMRI studies}

Despite the growing attention to the BST in animal research, human researchers have not yet fully embraced the notion of the extended amygdala, and few human neuroimaging studies discuss the role of the BST. Although brain imaging studies reporting amygdala activation number in the thousands, there are fewer than 100 human imaging studies examining the BST (Fig. 4). This discrepancy, in part, results from the fact that most available software for automated labeling of cluster locations does not include the BST. Thus, researchers lacking a background in comparative neuroanatomy may remain unaware that their cluster encompasses the BST region. Moreover, the BST's location, shape, and size make it difficult to clearly identify using MRI. Specifically, the BST is small and oblong, which, when combined with low-resolution imaging and common preprocessing techniques (e.g. Gaussian blurring), can leave the BST indistinguishable from bordering regions at the resolution of most brain imaging studies (often acquired in $2-4 \mathrm{~mm}^{3}$ voxels). Thus, even individuals familiar with the BST often remain cautious in claiming that their finding results from BST activation.

Researchers should continue to approach naming their clusters with caution, particularly because the BST nuclei are intermixed with other nearby regions, such as the shell of the nucleus accumbens. Nevertheless, regardless of the neuroanatomical label one chooses to apply to clusters that encompass portions of the BST, it remains clear that study of this region will reveal important new information relevant to understanding the neurobiology of emotion. Therefore, we recommend that, even in a situation where researchers cannot attribute an activation cluster specifically to the BST, researchers discuss the role of the BST as well as other basal forebrain regions that could give rise to the activation cluster. Additionally, we recommend that scientists specifically interested in human central extended amygdala regions begin to explore high-resolution MRI (e.g. [27]) and/or combine MRI with chemoarchitectonic PET imaging to precisely localize an individual subjects activation (e.g. [69]). By exploring the relationship between BST-region activation and the theorized role for the BST in threat processing, we will be able to link mechanistic studies in specific subnuclei to the experience of fear and anxiety in humans suffering from stress-related psychopathology. 


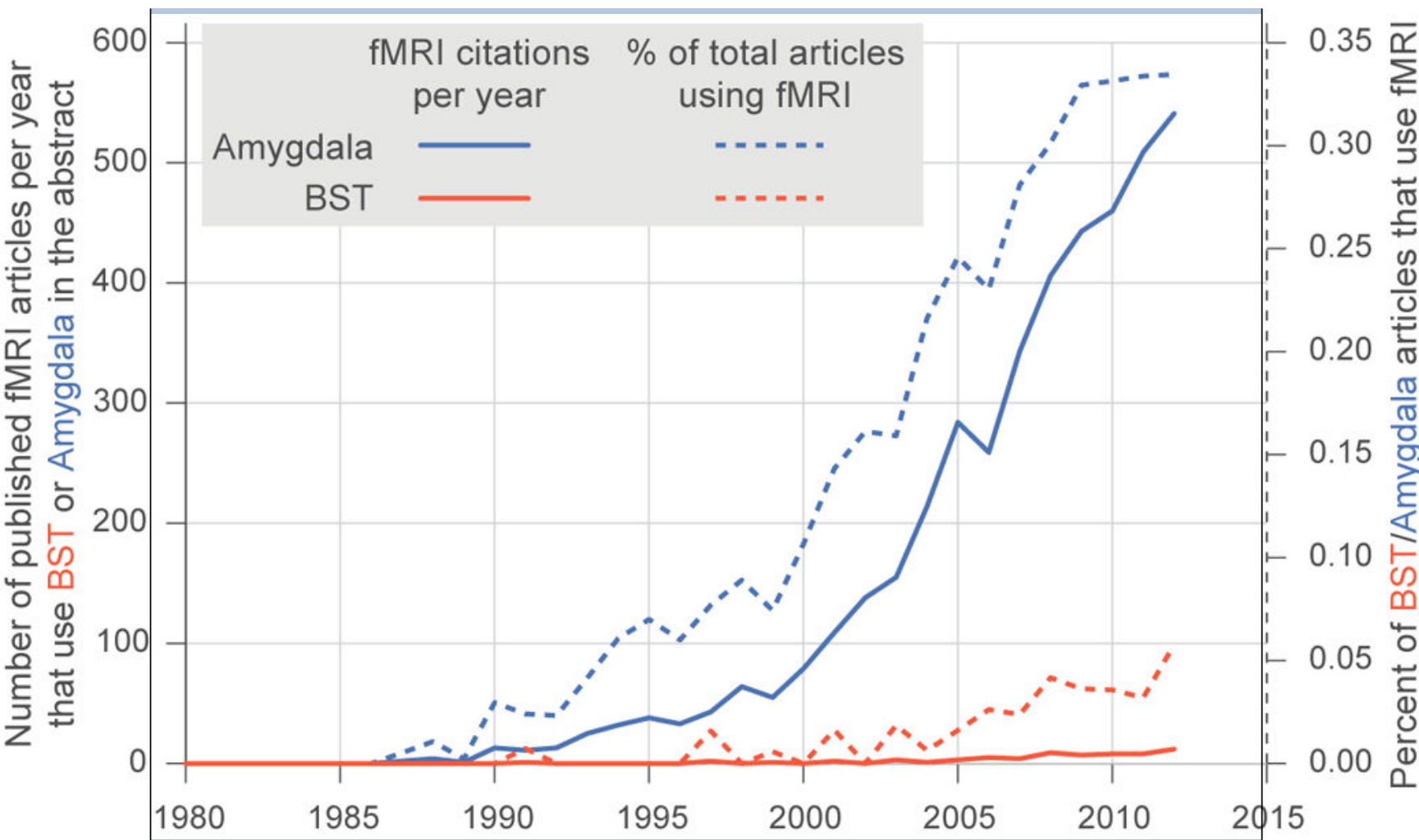

Figure 4. The number of fMRI articles calling attention to the "Amygdala" as compared to "BST" or extended amygdala

More specifically the total number of fMRI articles (solid) and percentage of total articles using fMRI (dashed), where pubmed searches matched: Amygdala (blue), and ("Extended amygdale" OR BNST OR BST OR "Bed Nucleus of Stria Terminalis" OR "Bed Nucleus of the Stria Terminalis") (red). 


\section{BOX 2: A broader role for the Extended Amygdala}

Here we have focused on the central extended amygdala's role in threat processing, and urge researchers to consider it in relation to models of human threat-processing and stress-related psychopathology. Nevertheless, it is important to emphasize that our focus on threat-processing in the central extended amygdala is primarily motivated by the extant literature, and that, 1) this is not the sole role of the central extended amygdala which also plays a role in appetitive behaviors, and 2) many other regions play a role in threat processing, including the medial extended amygdala.

1. The Extended Amygdala in Appetitive \& Addictive Behaviors. The extended amygdala plays an important role in appetitive [98] and addictive behaviors [16], including motivated feeding, and potentially hunting [99,100]. Via projections to reward-related brain regions (e.g. VTA), appetitive behaviors can be initiated and suppressed from the central extended amygdala, as well as from the sexually dimorphic medial extended amygdala. For example, stimulation of BST afferents in rodents can both induce and attenuate appetitive behavior, depending on the type of neuron being activated [101]. In humans, activation of the BST-region can reflect the trade-off between competing threats and rewards [102]. Moreover, the anterior edge of the extended amygdala includes portions of the shell of the nucleus accumbens, which contains neurons that, depending on the context, can code for either positive or negative information [103]. The data presented here, along with that reviewed elsewhere [104,105], suggest that the extended amygdala plays a broader role in balancing avoidant and appetitive motivations, which, when disrupted can form the core of many forms of psychopathology.

2. The role of other regions in the processing of threat. We propose that the central extended amygdala serves to link threat-related information with the appropriate behavioral and physiological outputs, which requires the coordinated function of a distributed brain circuit. Though this review is centered on the central extended amygdala, the importance of other parallel and complementary circuits cannot be understated - threat is not processed solely in the central extended amygdala (see: [15]). For example, in addition to the $\mathrm{Ce}$, the Me, which is part of the medial extended amygdala, has the capacity to initiate threat-responses via projections to hypothalamic and brainstem regions [106]. Moreover, rodent studies have demonstrated that the Me plays a role in predator induced threat responses [11,107-109]. To date these rodent findings, demonstrating that the medial extended amygdala parallels the central extended amygdala, have yet to be translated to primate species. Nevertheless, the medial extended amygdala exemplifies a parallel circuit that should also be considered in relation to the pathophysiology of anxiety disorders.

In addition to circuits that work in parallel, there are many complementary regions that influence and/or are influenced by the central extended amygdala. These regions include, but are not limited to prefrontal cortical regions that have the capacity to convey regulatory and evaluative information $[77,110,111]$, and hypothalamic and brainstem 
regions that are required to enact threat-responses $[19,112,113]$. Moreover, functional connectivity analyses in humans suggest that the central extended amygdala may be associated with increased coordination among disparate functional circuits during the processing of potential threat [114]. To establish causality, human and non-human primate studies should further examine the effects of brain lesions that selectively disrupt functions of the central extended amygdala and complimentary regions on distributed brain function during the processing of threats (e.g. following the work of: $[77,88])$. 


\section{Outstanding Questions}

- What is the role of the central extended amygdala in dispositional fear/anxiety and/or anxiety disorders?

- Do the human Ce and BST differentially contribute to the subjective feelings of fear and anxiety? Are they uniquely involved in responses to acute or potential threat?

- Do the Ce and BST differentially contribute to different clinical diagnoses and/or aspects of stress-related psychopathology? How can this information advance diagnoses and treatments?

- What functions are unique to the central extended amygdala, if any?

- How does the function of the central extended amygdala contribute to the balance between defensive and appetitive behaviors?

- What brain systems modulate central extended amygdala function?

- How do genes and environmental experience influence function within the central extended amygdala? Are its components differentially affected?

- How flexible are central extended amygdala circuits? Can they be altered with training or treatment? 

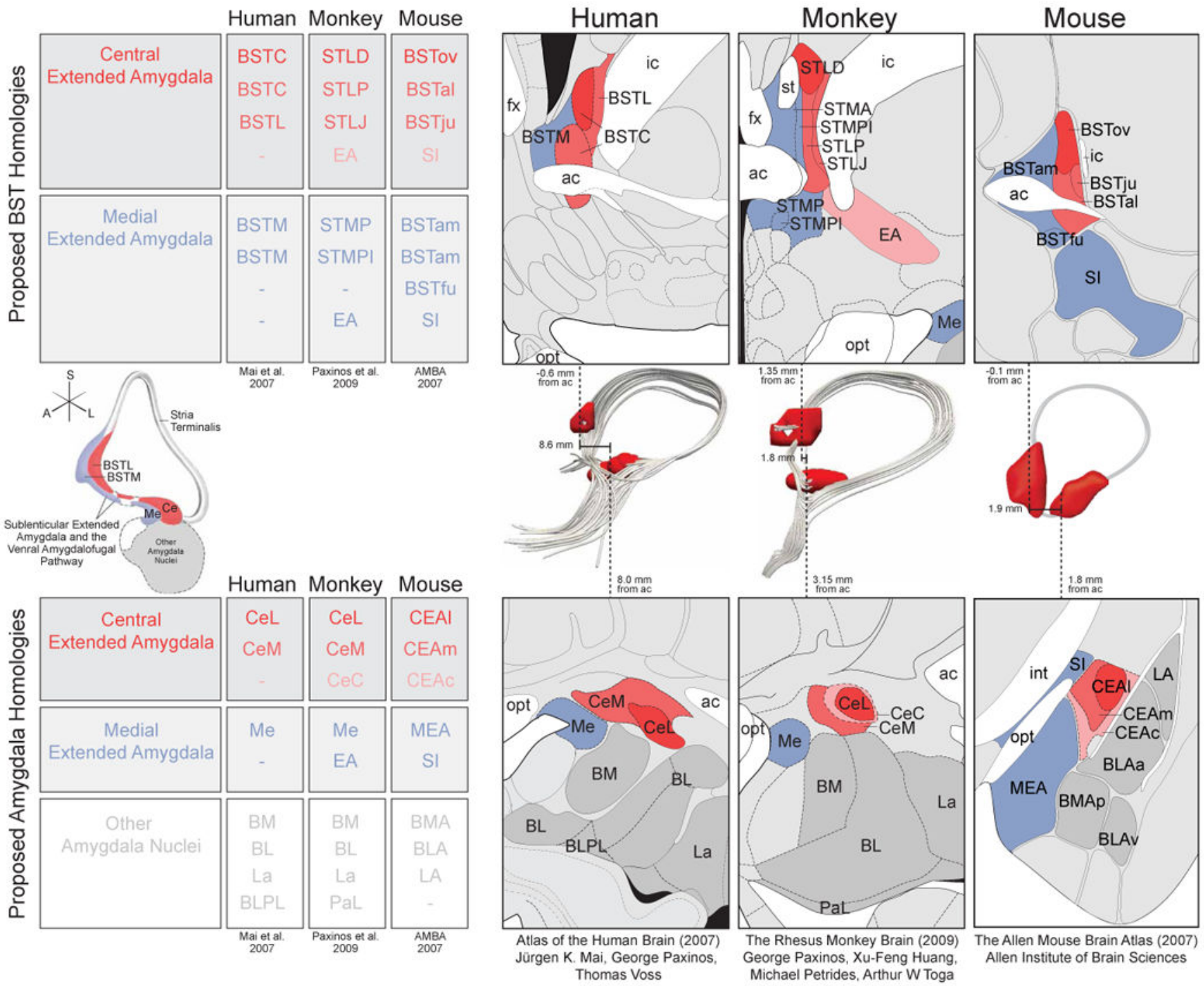

Figure 1. Central extended amygdala (red/pink) and medial extended amygdala (blue) both encompass portions of the BST (top), Ce-BST pathways (middle), and amygdala (bottom)

Although nomenclature varies by species, and by researcher, many regions are thought to have cross-species homology, which is denoted by color throughout and depicted in tables for BST (top left) and amygdala (bottom left). Atlas slices were adapted to highlight the proposed extended amygdala homologies across human ([115]; left), rhesus monkey ([116]; middle) and mouse ([35]; right). Ce-BST connections are show in 3D across the middle row, depicted with: a schematic from Heimer et al. ([117]; left), human DTI, rhesus monkey DTI, and a corresponding mouse drawing (right). Importantly, dashes in the tables do not always denote a lack of homology, but rather a lack of correspondence across atlases. For example, although the $\mathrm{CeC}$ can be seen in the human brain [117], this sub-region of the Ce is not included in the Mai et al. 2007 human brain atlas. All figures reprinted with permission. 


\section{a) $\mathrm{Ce}$ and BST sub-nuclei recieve projections from similar regions}

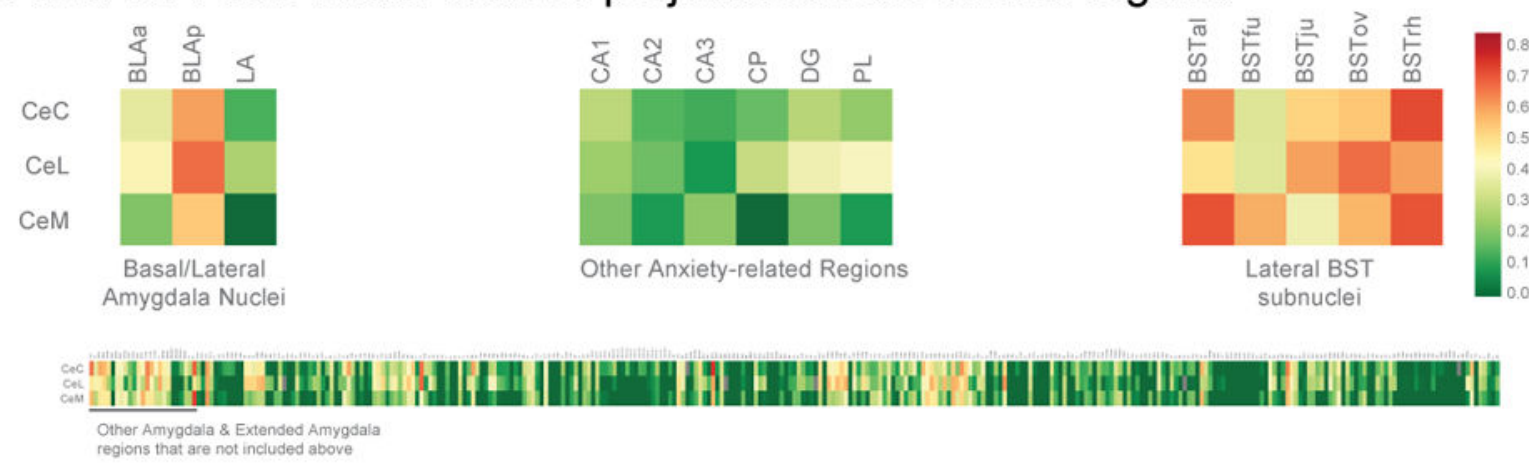

b) $\mathrm{Ce}$ and BST sub-nuclei project to similar regions
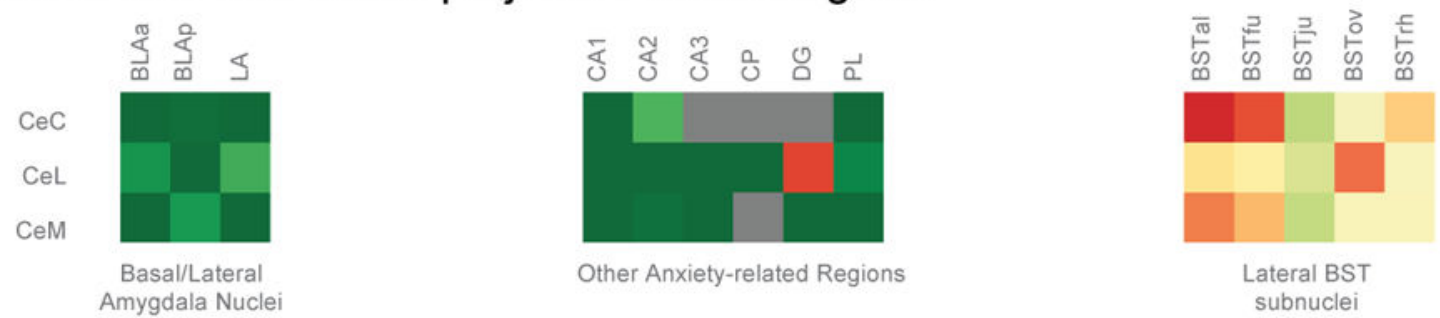

Other Anxiety-related Regions

Lateral BST subnuclei

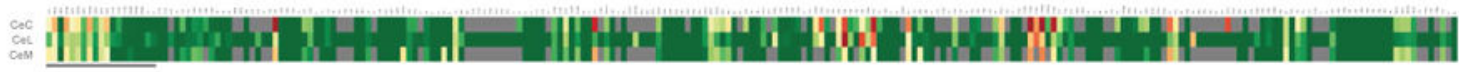 Other Amygdala \& Extended Amygdala
regions that are not included above}

c) Ce and BST sub-nuclei share similar gene expression patterns

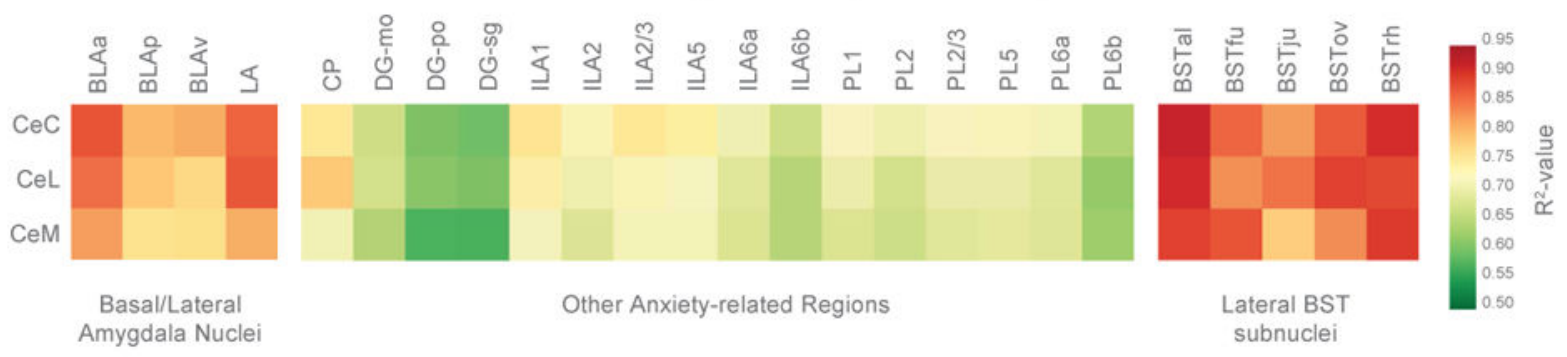

\section{ะ \\ Other Amygdala \& Extended Amygdala}

Figure 2. The Ce and BST show similar patterns of connectivity in rats (a \& b), and similar gene expression in mice (c)

We identified those brain structures that most often projected to, or received projections from, the same regions as the individual $\mathrm{Ce}$ sub-nuclei $(\mathrm{CeL}, \mathrm{CeM}$, and $\mathrm{CeC})$. To accomplish this, we extracted information about efferent and afferent connectivity strength (ranging from does not exist to very strong) for all available brain regions. The efferent and afferent connectivity patterns for each region were correlated with the respective patterns of connectivity for the Ce subnuclei. Because the database is incomplete and we used correlational statistics, we restricted correlations to those based on at least 20 data-points. 
Results demonstrated that BST subnuclei shared similar inputs (a) and outputs (b) with Ce subnuclei, as compared to other amygdala nuclei (top left), other anxiety-related regions (top middle), or the rest of the brain (bottom). To understand how gene expression within the $\mathrm{Ce}$ relates to that in the BST, and the exclusivity of this relationship, we performed a large-scale analysis with the Allen Institute for Brain Science (AIBS) atlas of mouse gene expression [35]. Specifically, we examined the correlation between gene expression profiles in $\mathrm{Ce}$ subnuclei with gene expression profiles in other brain regions. Results demonstrated BST subnuclei to have similar gene expression profiles to Ce subnuclei, as compared to other amygdala nuclei (top left), other anxiety-related regions (top middle), or the rest of the brain (bottom). For details of the relationships between Ce subnuclei and other brain regions, zoom in on the online version to read text in the bottom panels. 


\section{a) Indivıdual non-human prımate ımagıng studies}

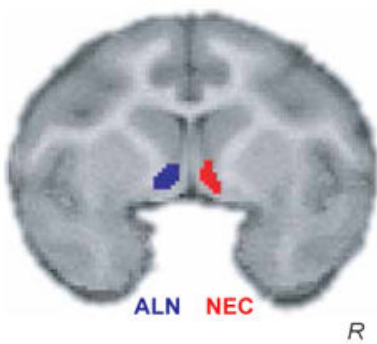

Kalin et al., 2005

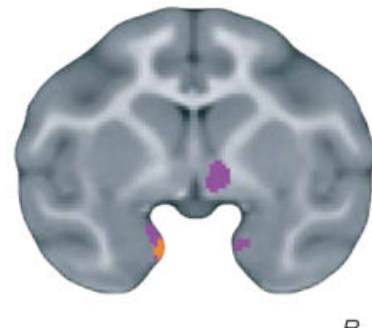

Fox et al., 2008

b) Indıvıdual human ımagıng studies

c) Conjunction of curated meta-analyses of negative affect and fear conditioning

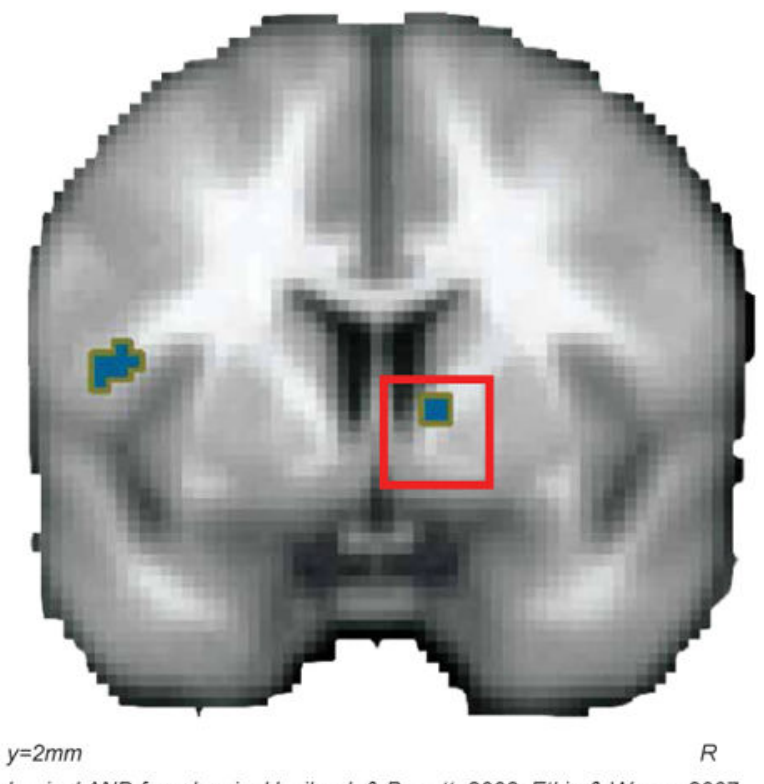

Logical AND from Lewis, Haviland, \& Barrett, 2008; Etkin \& Wager, 2007

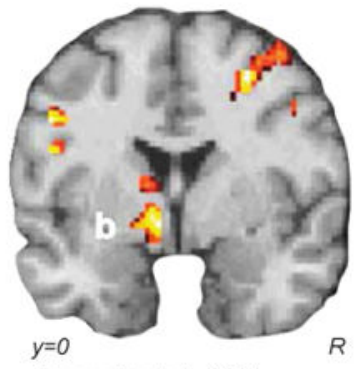

Somerville et al., 2010

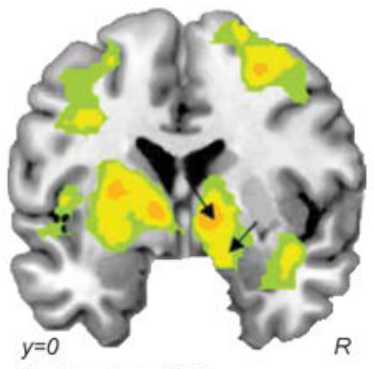

Mobbs et al., 2010

d) Automated meta-analysis of "anxiety"

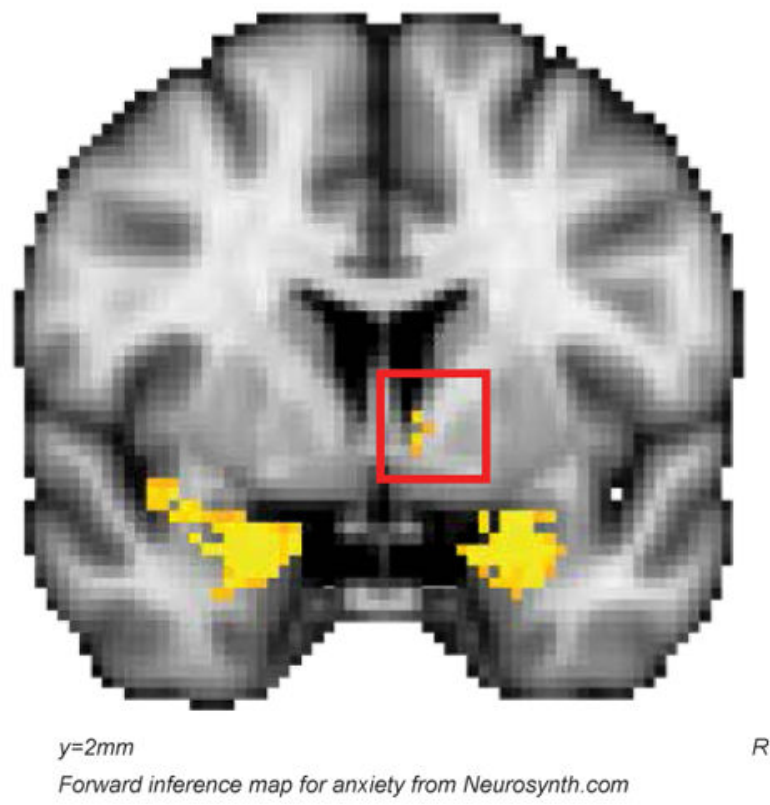

Figure 3. Individual neuroimaging studies in non-human primates (a) and humans (b) as well as meta-analyses of human neuroimaging data (c-d) demonstrate BST involvement anxiety-related behavior and prolonged threat preparedness

In non-human primates individual differences in brain metabolism in the BST-region were correlated with variation in freezing (a, left) while animals were alone experiencing separation stress (ALN; blue) as well as during exposure to a potentially threatening human intruder making no-eye-contact (NEC; red); and variation in anxious temperament (a, right) during both ALN \& NEC (purple). In humans, fMRI activation in the BST-region while potential threats were getting closer, as participants were earning shocks (b, left) or as a spider was approaching their foot ( $b$, right). A Logical AND conjunction ( $\mathrm{p}<.005)$ of curated meta-analyses of human neuroimaging studies examining 1) the experience of negative affect, and 2) threat conditioning in anxiety-disorder patients compared to controls reveal activation in the BST region (c). In addition, an automated meta-analysis using Neurosynth [92] found that the 222 brain imaging papers using the word "anxiety" were more likely to report activations in coordinates near the BST in comparison to all of the papers in the database (d). Although we do not interpret these studies as conclusive evidence for 
involvement of the central extended amygdala in anxiety, together these studies provide the necessary initial data to motivate further study of this region in the primate brain. All figures were reprinted with permission. 